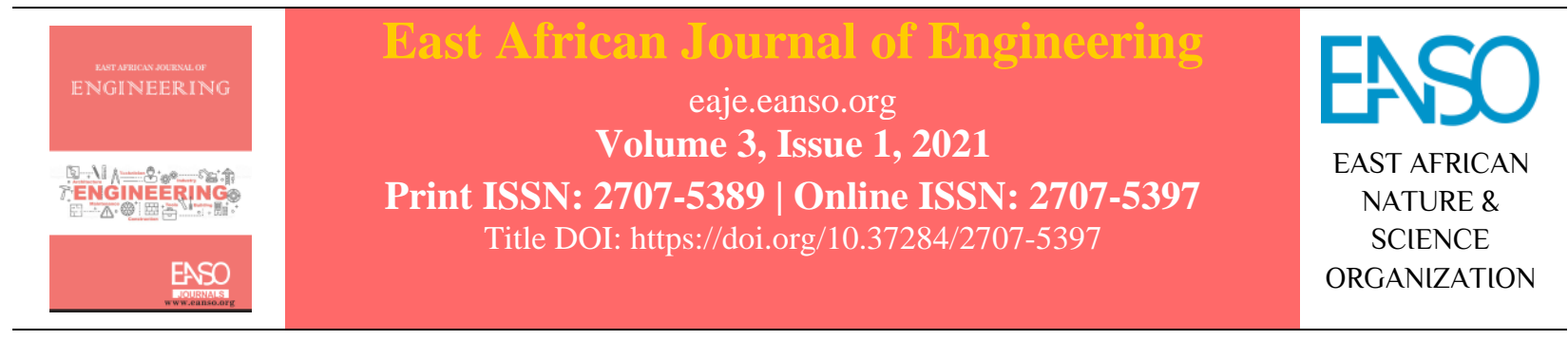

Original Article

\title{
Developing Workshop Safety Management Skills for Kyambogo University Mechanical Production Engineering Students in Uganda.
}

\author{
Peter Agole ${ }^{I^{*}}$ \& Wilson Okaka, PhD \\ ${ }^{1}$ Kyambogo University, Kyambogo, P. O. Box 1, Kampala, Uganda. \\ * Author for Correspondence Email: aapalaomyek@gmail.com.
}

Article DOI: https://doi.org/10.37284/eaje.3.1.416

\section{Publication Date: ABSTRACT}

21 September 2021 Effective workshop management skills are vital for the occupational safety of the students, lecturers, instructors, support staff, or technicians who use mechanical

Keywords: production or manufacturing workshops at universities. The main purpose of the paper is to examine the status of the undergraduate university students' skills

Vocational practice in safety management in the engineering educational workshops assess

Engineering, the common occupational hazards and risks in the workshops, and explain the

Mechanical prospects of promoting occupational safety standards. The paper was informed by

Engineering, a recent study that was done in Kyambogo University, Uganda with a focus on

Vocation, practice, implement safety measures for skills practice in the department of

Workshops, mechanical production Engineering. In the study, a descriptive research design

Uganda. was used, where both qualitative and quantitative research methods were used. The total number of respondents who participated in the study was 30 . The respondents included the head of the department, lecturers, students, technicians from the Mechanical Production Engineering Department of Kyambogo University. Data were collected from respondents through the use of questionnaires and interview guides. Data were analyzed and presented using tables and then descriptions were done using percentages. The findings revealed the following occupational hazards and risks, physical hazard, ergonomic hazards, chemical hazards, psychological hazards, and biological hazards. The study also revealed that accidents always occurred in the department of mechanical and production workshops. The major causes of the accidents were due to lack of knowledge or skills, safety policy gears, carelessness, safety protective wears, proper storage of materials, and instructions' manuals or guidelines. In addition, there are problems of lack of modern tools, digital technology, good supervision, adequate space, and awareness of the ICT led safety systems. The cost-cutting strategies for workshop safety management were warning signs, alerts, and clear labels, use of protective gear, providing first aid kits, vocationalised training, and awareness campaigns on occupation safety. There is a need for routine monitoring,

114 | This work is licensed under a Creative Commons Attribution 4.0 International License. 
renovations, replacing obsolete machines, safety tags, fire drills, industryuniversity benchmarking, or industrial placements.

\section{APA CITATION}

Agole, P., \& Okaka, W. (2021). Developing Workshop Safety Management Skills for Kyambogo University Mechanical Production Engineering Students in Uganda East African Journal of Engineering, 3(1), 114-122. https://doi.org/10.37284/eaje.3.1.416

\section{CHICAGO CITATION}

Agole, Peter, and Wilson Okaka. 2021. "Developing Workshop Safety Management Skills for Kyambogo University Mechanical Production Engineering Students in Uganda". East African Journal of Engineering 3 (1), 114-122. https://doi.org/10.37284/eaje.3.1.416.

\section{HARVARD CITATION}

Agole, P., \& Okaka, W. (2021) "Developing Workshop Safety Management Skills for Kyambogo University Mechanical Production Engineering Students in Uganda", East African Journal of Engineering, 3(1), pp. 114-122. doi: 10.37284/eaje.3.1.416.

\section{IEEE CITATION}

P., Agole., \& Okaka, W. "Developing Workshop Safety Management Skills for Kyambogo University Mechanical Production Engineering Students in Uganda," EAJE, vol. 3, no. 1, pp. 114-122, Sep. 2021.

\section{MLA CITATION}

Agole, Peter, \& Wilson Okaka. "Developing Workshop Safety Management Skills for Kyambogo University Mechanical Production Engineering Students in Uganda." East African Journal of Engineering, Vol. 3, no. 1, Sep. 2021, pp. 114-122, doi:10.37284/eaje.3.1.416.

\section{INTRODUCTION}

Over ten years of the corresponding author's stay in the department of mechanical engineering, several accidents have occurred on the young generation of engineers. Despite the occurrences of accidents, no emphasis has ever been put to improve the safety management skills of students in this workshop neither has any research been done to find out the causes of the mayhem in the workshop. Following the author's observation of the situation state of accidents in the workshops, there was a wide gap in knowledge hence the need for action research in the department to propose was to solve the problems in the workshop.

According to the World Health Organization (WHO), each year an estimated 100 million workers are injured, 200,000 die each year in occupational accidents and 68 million to 157 million new cases of occupational disease are attributed to hazardous exposures in workplaces. The high numbers of adverse health outcomes impact significantly on the health of the world's population. Occupational injuries and diseases have profound effects on work productivity and on the economic and social wellbeing of workers, their families, and dependents (Veltri, 2011). Since 1995, the International Labour Organization (ILO) and the World Health Organization (WHO) have shared common objectives of occupational health. It was adopted by the Joint ILO/WHO Committee on Occupational Health at its first session in 1950 and revised at its twelfth session in 1995. The objectives were maintenance and promotion of workers' health and working capacity, improvement of working environment and work to become conducive to safety and health. In addition, they also jointly shared an objective on the, development of work organizations and working cultures in a direction, which supports health and safety at work and in doing so promotes a positive social climate and smooth operation and may enhance the productivity of the undertakings.

According to Viscusi (2008), statistics in Ugandan industrial sector, the manufacturing sector was the most hazardous in that accounted for most occupational fatalities with $84 \%$ of the occupational fatalities and only $3 \%$ of the occupational injuries, 
followed by the construction sector with $12 \%$ of the occupational fatalities and $29 \%$ of the occupational injuries. The service industry had $3 \%$ of occupational fatalities and $56 \%$ of the occupational injuries. Other sectors accounted for only $1 \%$ of the occupational fatalities and $12 \%$ of occupational injuries. In 2002, the number of occupational accidents increased by $69 \%$ as there was 354 occupational accidents in Kampala resulting in death or serious injury that required more than two days of absence from work. The reported occupational accidents in 2002 resulted in 34 occupational fatalities, a $63 \%$ reduction in the number of deaths, compared to the previous year (Paton, 2008).

Narrowing to Kyambogo University, there are very many challenges that affect both staff and students at the department of mechanical and production engineering (DMPE) at the university. Some of the challenges include but not limited to poor lighting in the mechanical workshop, broken windowpane, absence of instructional and or prohibition signage, absence of machine guards, warning signs to guard against accidents spillage of coolant on floor which causes users to slip and fall, lack of water for washing hands after work, poor storage of materials and tools including lack of gang-ways in the workshops. These problems prompted this study to improve general health and safety practices at the machine workshop in the department. In 2006 February, one student of environmental engineering cut her finger with the shearing machine during practical in DMPE Kyambogo University. Therefore, for developing and improving the workshop safety management skills for DMPE the university. The study aimed at identifying safety measures to be used in skills practice designing precautionary signs for skills practice and implementing safety measures for skills practice in the department of mechanical and production engineering.

\section{LITERATURE REVIEW}

There is a need for safety in the mechanical and production engineering workshops, according to Ugandan Ministry of Gender, Labour, and Social Development (2015), when hazards cannot be removed in workshops or replaced with less hazardous alternatives, the next best control option is the enclosure. Enclosing a hazard usually means employees are not exposed to it during normal operations, although they could be during maintenance or if the enclosure system breaks down. For these situations, consider additional controls such as specific work procedures or personal protective equipment (PPE) (Diving Regulations, 2009). Remove, replace, or enclosing of a potential hazard as well as installing a barrier to the exposure are advocated by PPE. In the case of air contaminants, local exhaust ventilation systems should be installed to remove the contaminants (Diving Regulations, 2009). However, barriers to the ventilation systems may include ventilation hoods (fume cupboards) in laboratories, machine guarding including electronic barriers, isolating processes in areas away from workers, except for maintenance work, baffles used as noise-absorbing barriers and heat shields (Lebergott, 2002).

In addition, there is a vital need for minimization using administrative controls in workshops. As Viscusi (2008) noted, administrative controls use procedures or instructions to minimize exposure to risks could limit the exposure time to a particular hazard such as noise or radiation. According to Paton (2008), if plant or equipment failure in your workplace has the potential to cause injury or illness, you need an effective system for properly maintaining the plant and equipment. In relation, Kyambogo University provides various equipment that helps to protect students from exposure to hazards (Health and Safety Executive 2009). Diving Regulations (2009) asserts that it is important for administrations to make sure they support any new control measures with work procedures with maintenance being an important part of the process.

116 This work is licensed under a Creative Commons Attribution 4.0 International License. 
Paton (2008) noted that PPE is used to reduce or minimize exposure to and contact with agents that cause physical, chemical or biological injuries.

Besides, legal requirements are required for wellregulated safety standards at workplaces. In Kyambogo University mechanical workshops, sometimes students and workers provide their own protective means like protective glasses to help them reduce exposure to workshop risks. Appropriate safety measures are recommended for every safe workplace in workshops. Specific safety, efforts should target safety culture improvement and employees should beheavily involved in these efforts. This helps increase personal responsibility and employees' compliance with safety standards (Geller, 2005). Furthermore, (Bruce, 1995) asserts that risk management operates beyond occupational hazard precautions when calculating the victims risks and benefits. Its response is based on the standard application of broader control strategies, which may include rehabilitation and claim management to mention a few.

Compliance with legal requirements is aimed at attracting cases at opposite ends of the spectrum (Stricoff 2000) asserts that while compliance with legislation underpins safety management system and stimulates hazard identification and assessment project at the auto press, in pig works conforming to legal requirements appears synonymous with minimum safety effort. Hence, safety management has to be held personally responsible for safety. That means everyone in a company/institution can be held accountable (Garner \& Horn, 2000). In successful businesses, leaders are graded not only on a financial score card but also on their ability to integrate safety into the business process (Krause, 2000). Safety is a core value, which must be integrated into the day-to-day operations of the business. Employee engagement in safety management is key stemming from CEO down to the least paid employee. Krause (2000) notes that every employee needs to engage in the safety management process because failure inhibits the potential of becoming a world-class company or institution in safety or in business. Therefore, in cases where management is detached from safety management policies, the employees tend to ignore safety practices at the workplace. Noteworthy, whenever safety practices are followed properly, they prevent most work-related accidents hence provides a better working environment for interaction with personnel and equipment for better production.

Lee (2009) asserts that safety should not be an emotional subject any more than anything else in business should. He suggests devoting energy to finding the root cause of anything that goes wrong in a disciplined way. What gets investigated are usually the symptoms of the problem when the real problem is the culture in a related issue of treating a symptom instead of the root cause. Eckenfelder (2000) notes that the root cause of the fatal Columbia shuttle crash that was perhaps one of the root causes of additional safety standards culture by NASA.

Furthermore, the impact of safety management systems is significant. Investigation of the links between safety management effort and injury performance also connects a group of studies, which analyze safety program effectiveness. The studies have explored organizational and health and safety system characteristics sometimes referred to as the 'safety climate' (Glennon, 2011). Viner (2012) asserts that among the ways of managing safety, there is the audit review: the audit criteria call for the conduct of planned and documented safety management systems to be undertaken by competent persons, with reports distributed to appropriate personnel in the organization. The deficiencies identified and corrected. In turn, audits provide a tool for the formal review at the senior level of the appropriateness and effectiveness of the safety management system. This can effectively be executed using Kolb's model of training /working 
Figure 1: Shows a demonstration of Kolb's experiential Cycle

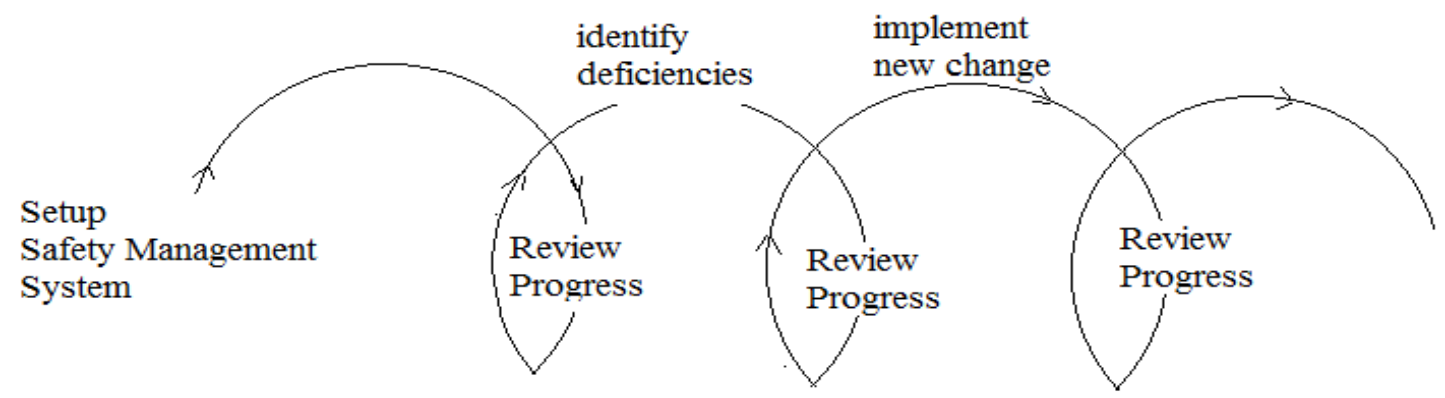

Source: (Swartz, 2003)

According to Lebergott (2002), according to the audit criteria, health and safety responsibilities should be defined and communicated in enterprise policies or directives, job descriptions or manuals. Clear authority must be allocated for ensuring current legislation is available and for the identification and allocation of responsibilities imposed by legislation in addition to ensuring the responsibility are consistent with legislated statements. Build a shop uses the site safety plan for each construction project to outline the responsibilities of specified positions. There is less vigilance across the cases towards the identification and allocation of legislative responsibilities ensuring managers and supervisors are aware of the legislative responsibilities towards contractors and the provision of information more generally on legal responsibilities. Paton (2008) asserts that the audit criteria for effective line management and supervisor involvement envisage for an active and broad-ranging role for the line manager and supervisor. These include the involvement in planned hazard management activities, health, and safety consultative arrangements, more traditional activities such as incident investigation, and other activities identified. Supervisors are expected to be trained appropriately in order to participate effectively in hazard management and to incorporate health and safety into daily supervision of work.
According to Paton (2008), eating and drinking in the mechanical workshop areas is strictly prohibited. When working with machine tools or other equipment with rotating spindles, jewelry, loose clothing, etc. are prohibited and long hair must be completely covered. Personal protective equipment (PPE) is often supplied and must be used where necessary. Barrier cream, lab coats/overalls, eye and hearing protection, dust masks and safety shoes must be used as the work/risk assessment dictates, Lab coats/overalls and safety boots must be worn by technicians operating the machines. The gangway through the workshop must be kept clear. Any oil spillage and grease must be cleaned up immediately. Workshop users are also encouraged not, to carry loads as they can be dangerous or vision obscured.

WHO (2014) notes each safety signboard and associated supplementary signboard should be used to convey only one safety message. Composite signs giving more than one safety message are prohibited. Graphical symbols should not be combined to convey more than one safety message. For example, if a mandatory instruction to wear safety helmets and safety goggles is required two signboards should be used. The safety helmet and safety goggles should not be combined as one graphical symbol. All portable electrical equipment must be regularly inspected and tested for electrical safety. Lifting equipment must be registered with the University's Engineering Insurers and be subject to

118 | This work is licensed under a Creative Commons Attribution 4.0 International License. 
annual inspections. All the air receivers must be subject to a thorough examination at the statutorily required intervals, where applicable suitable Local Exhaust Ventilation (LEV) must be used (Goodson, 2015).

\section{METHODOLOGY}

The descriptive research design was adopted for this study. Qualitative method of data collection was employed. The target study population comprised of the head of the department, lecturers, and students in the DMPE. Students were selected using a simple random sampling method so as to give each of them an equal chance of being selected. A sample of 45 respondents was obtained from the staff and students of the department. The study adopted purposive sampling to select the head of the department, lecturers, and technicians. A structured interview guide was used to collect information from users of the workshop to obtain firsthand information. This helped me obtain information from the students, lecturers and technicians while at work. The questionnaire used to collect data was pretested on few respondents from the DMPE to check if they are valid in capturing the information that is needed for the research. Interviews and documents were then reviewed according to Kolb's experiential cycle.

Raw data collected was first sorted, edited, coded and then entered into a computer spreadsheet and analyzed using Microsoft Excel (spreadsheet). The response rate for each item in the questionnaire and site observation datasheet was analyzed. The analyzed data was finally presented using descriptive methods for easy interpretation and enable comparisons and inferences to be drawn. This was supplemented with qualitative data obtained in the form of verbal responses from interviews.

\section{RESULTS AND DISCUSSION}

Respondents were asked if they had experienced or heard of any students who having been ever exposed to the occupational hazards at the university departmental workshop. Findings revealed that most of the respondents (70\%) had experienced or heard of their friends who were exposed to occupation hazards, (17\%) reported being exposed to occupational hazards, and (13\%) declined to volunteer any information on the subject. In another development, the respondents were asked to recall the common hazards in the university departmental training workshop. Among the hazards that occur, physical hazards were commonest (47\%), followed by psychological hazards (20\%), chemical hazards $(13 \%)$, ergonomic hazards (13\%), and biological hazards $(7 \%)$.

The majority of respondents agreed that accidents rarely occur in the department $(63 \%)$, while $27 \%$ of the respondents claimed the accidents occurred frequently and $10 \%$ agreed that accidents seldom occur there. In regard to the common causes of accidents in the department, the study showed that the common causes were due to lack of knowledge about tools used and equipment used in the workshop (23\%). $20 \%$ of the respondents attributed causes to lack of safety gears while $17 \%$ to the carelessness of students. $10 \%$ reported nonuse of safety wears as well as to nonuse of safety protective wears. 7\% reported a lack of awareness of the operations safety hazards, $7 \%$ attributed them to the lack of proper instructions to follow. $7 \%$ also reported the use of wrong tools for specific tasks, $3 \%$ said it was caused by a lack of supervision, congestion, and improper storage system for the workshop materials. Results indicated that $73 \%$ of the respondents had ever been exposed to risks in the workshop and only $27 \%$ of the respondents had never been exposed to risks. On the issue of first aid facilities in the workshop, the findings indicated that $70 \%$ of the respondents that there were first aid facilities in the department and only $30 \%$ said that there were no first aid facilities. In addition, when respondents were asked about the facilities that should be put in the training workshops, results revealed that $40 \%$ of the respondents recommended 
for gangways to be put in the training workshop $27 \%$ advocated for proper lighting, $13 \%$ for machine guards $13 \%$ of the cited a need for first aid kit and finally, $7 \%$ agreed to have a good drainage system.

When asked about the possible ways of improving the department workshop $20 \%$ of the respondents suggested that there should be warning signs $17 \%$ recommended for the provision of protective gear to students and staff (any workshop users) while 3\% called for the provision of first aid kit. The other $13 \%$ said that there is a need to educate and train people about the workshop injuries, $10 \%$ asked for health and occupation safety improvement, renovation of workshops, early warning tags on machines, and the need to subsidize industrial growth.

The results of the study on the occupational health hazards in DMPE showed that there were some obvious unsafe working conditions. It showed that there were a number of categories of insidious hazards. These included physical hazards such as noise, vibrations, unsatisfactory lighting, radiations and extreme temperatures, explosions, and falling bodies. Hazards associated with non-application of ergonomic principles included poor mechanically conditioned machinery; poorly designed mechanical devices and tools used by workers improper seating bad workstation design and poorly designed work practices. Injuries were the highest identified with a total of 8 injuries followed by diseases and accidents. In 2010 ordinary Diploma students in Mechanical and Production crushed his hand in a drilling machine at DMPE Kyambogo University. In 2012, two students crushed their hands in sheet metal rolling machines while in 2015, another student slipped and fell on the lather machine which is without a guard. A welding machine caught fire also in 2015 , which caused huge financial loss to the university in order to replace it with a new machine.
It was found out that lack of knowledge about tools used and equipment was the major cause of accidents in the mechanical and production department. It was revealed that some workers and students in the department did not have enough knowledge about the machines; as a result, they mishandled them and end up into accidents in the workshop. A respondent said that; "we lack enough training on machine usage and this has increased accidents within the workshop". This is in the same line with Veltri (2011) who says that inadequate knowledge on machine usage is one of the biggest challenges that cause accidents in workshops. The carelessness of students was also given as a reason for accidents in the workshops, this is because some students tend to mishandle the machines and on top of that the machines have no warning signs and hence resulting in accidents in the workshops. This finding agrees with that of Morrison (2013) who noted that the major cause of accidents in the workshop is the carelessness of the employees. Therefore, safety could be observed and practiced in the mechanical and production engineering workshop at Kyambogo University, which accounts for the largest number of graduate engineers in Uganda.

Regarding the facilities needed for training in mechanical and production department workshops, the study also revealed that there is a need to put machine guards in the department. According to respondents, these machine guards help to prevent injuries in the department. During the interview with respondents, a respondent said that;

"The administration should put machine guards within the workshop and this will help reduce on the injuries within the workshop"

In the same line, Petersen (2000) in his findings of the study conducted in 1997, he found out that machine guards can help reduce accidents in the engineering workshops. It was revealed that there is a need for the provision of the health team and the first aid kit in the department. There should be 
policy/procedure development, health and safety planning, measurement and review, and planned hazard management programs, together with health and safety promotional activities and this will help to reduce injuries in the department.

\section{CONCLUSIONS AND RECOMMENDATIONS}

The study found that the safety conditions of the educational workshops and laboratories at the university require considerable improvements. the department faced several basic problems such as lack of knowledge about tools used and equipment, carelessness of students, lack of supervision, nonuse of safety protective wears, lack of proper instructions to follow, use of unrecommended tools for specific tasks, over congestions, improper storage of materials, lack of safety gears and standards, and poor awareness of the fundamental operations safety hazards.

In addition, the investigation found that Kyambogo University seriously lacks the required proper standard and basic safety management practice training without any measures to improve them.

It is highly recommended that all the students, instructors, technicians, lecturers, visitors, and support staff in the department should be familiar with all the basic safety rules, regulations, and procedures related to the workshop operations and culture at the university.

There is a need to conduct a regular assessment, monitoring, evaluation, and improvement of compliance standards with the vital workshop safety rules in tandem with regular and periodic job safety analysis.

It is essential to monitor each and every operation for getting success, hence, all the processes and safety aspects must be monitored. Closely monitoring enhances the effectiveness of all safety tools on a regular basis and getting deviation or lapses, early cause detection and the remedial measures are recommended for immediate implementation.

The university should provide personal protective equipment to all employees within the plant. Equipment like gloves, overalls, and safety shoes, and safety helmets should be provided and can be replaced on old wear as immediate as possible.

It should be made mandatory to put on protective gear when the workers are within the plant. This will ensure that all injuries are prevented and by reducing exposure to risks. One of the reasons for poor safety was the failure to induct workers on safety upon joining the company. Induction of all new workers and visitors to the company should be mandatory to ensure that everyone is aware of the safety practices when carrying out the various tasks.

\section{REFERENCES}

Bruce, T. F. (1995). Occupational safety and health, small-scale enterprise and employment. African Newsletter on Occupational Health and Safety, 2(27).

Diving Regulations. (2009). The South African Occupational Health and Safety Act, 1993. Pretoria, SA: Government Printer.

Goodson, W. H., Lowe, L., Carpenter, D. O., Gilbertson, M., Ali, A. M., de Certain Salsamendi, A. L., ... Hu, Z. (2015). Assessing the carcinogenic potential of low-dose exposures to chemical mixtures in the environment: The challenge ahead. Carcinogenesis, 36, S254S296. https://doi.org/10.1093/carcin/bgv039

Health and Safety Executive. (2009): A Guide to Safety and Health Regulation in Great Britain. London, UK: Health and Safety Executive.

Lebergott, S. (2002)."Wages and Working Conditions". In David R. Henderson (ed.). Concise Encyclopedia of Economics (1st ed.). Indianapolis, Indian: Library of Economics and Liberty.

121 This work is licensed under a Creative Commons Attribution 4.0 International License. 
Ministry of Gender, Labour, and Social Development (2015): Home page". Www.empleo.gob.es. Retrieved 16 December 2015 from the following website: https://mglsd.go.ug/

Paton, Paton, N. (2008). Senior managers fail to show competence in health and safety. Occupational Health \& Wellbeing, 60(3), 6.

Swartz, G. (2003). Incident Reporting. Professional Safety, 38 (12), pp 32-34.

Veltri, A. (2011). Transforming Safety Strategy and Structure. Occupational Hazards, pp. 149-152.

Viner, D. (1991). Accident analysis and risk control.VRJ Delphi

Viscusi, W. K. (2008).Job Safety.In David R. Henderson (ed.), Concise Encyclopedia of Economics(2nd ed.). Indianapolis: Library of Economics and Liberty.

World Health Organization conference, (WHO, 1946. Www.empleo.gob.es. Retrieved 16 December 2015 